\title{
HARMONIC DIFFEOMORPHISMS OF THE HYPERBOLIC PLANE
}

\author{
KAZUO AKUTAGAWA
}

\begin{abstract}
In this paper, we consider the Dirichlet problem at infinity for harmonic maps between the Poincaré model $D$ of the hyperbolic plane $\mathbb{H}^{2}$, and solve this when given boundary data are $C^{4}$ immersions of $D(\infty)$, the boundary at infinity of $D$, to $D(\infty)$. Also, we present a construction of nonconformal harmonic diffeomorphisms of $D$, and give a complete description of the boundary behavior, including their first derivatives.
\end{abstract}

\section{INTRODUCTION}

Not much is known about nonconformal harmonic diffeomorphisms of $D$, the Poincare model of the hyperbolic plane $\mathbb{H}^{2}$ of constant negative curvature. A class of these harmonic maps can be obtained by lifting to the universal covers the harmonic diffeomorphisms between closed Riemannian 2-manifolds of constant negative curvature with different conformal structures [13, 18]. Another class is given by the Gauss maps of entire spacelike nonumbilical constant mean curvature surfaces in the Minkowski 3-space. $\mathbb{L}^{3}$, provided these surfaces are conformally equivalent to the unit disk and their lightlike sets coincide with the unit circle $[8,20]$. In this respect, Choi and Treibergs [7] constructed an explicit one-parameter family of nonconformal harmonic diffeomorphisms of $D$, each of which is realized as the Gauss map of an entire spacelike constant mean curvature surface of revolution in $\mathbb{L}^{3}$, and describes completely their boundary behavior (see also $\mathrm{Li}$ and Tam [16]).

Motivated by these results we shall consider a Dirichlet problem, the asymptotic Dirichlet problem, for harmonic maps between $D$ with $C^{0}$ boundary data from $D(\infty)$, the boundary at infinity of $D$, to $D(\infty)$. In this problem when constructing harmonic maps between $D$ with unbounded image, a main technical difficulty arises in obtaining a priori growth estimates for them. However, we will prove in $\S 3$ that this difficulty can be resolved under a suitable assumption on the boundary data, and then this asymptotic Dirichlet problem can be solved. In our construction of harmonic maps between $D$, we first construct a suitable barrier map at the boundary $D(\infty)$ for each boundary map which is assumed to be a $C^{4}$ immersion between $D(\infty)$. Then a priori growth estimates will be established in Lemma 5 with the aid of their barrier maps. In the course of proof we also present a construction of nonconformal harmonic diffeomorphisms of $D$, and give a complete description of the boundary behavior,

Received by the editors February 26, 1990 and, in revised form, February 6, 1992.

1991 Mathematics Subject Classification. Primary 58E20; Secondary 53C42, 53C50.

Partially supported by the Grant-in Aid for Scientific Research No. 01740075. 
including their first derivatives. We would like to point out that using the heat flow method, a general theory for the existence of harmonic maps with bounded energy density between complete noncompact manifolds was developed by $\mathrm{Li}$ and Tam [15]. Applying this result to the Poincaré model $D^{m}$ of the hyperbolic $m$-space $\mathbb{H}^{m}$, they also solved independently the asymptotic Dirichlet problem from $D^{m}$ to $D^{n}$ (for all $m, n \geq 2$ ) when given boundary data are $C^{3}$ maps of $D^{m}(\infty)$ to $D^{n}(\infty)$ with nonvanishing energy density. Recently, they [16] also obtained two fundamental results for uniqueness and regularity properties of proper harmonic maps from $D^{m}$ to $D^{n}$. In particular, the above smoothness assumption on the boundary data can be relaxed from $C^{3}$ to $C^{1, \alpha} \quad(0<\alpha \leq 1)$ (for further developments see [21]).

As an application of our method, we shall construct, in $\S 5$, entire spacelike constant mean curvature surfaces $M$ in $\mathbb{L}^{3}$, whose Gauss maps are harmonic diffeomorphisms of $M$ to $\mathbb{H}^{2}$ with $C^{4}$ diffeomorphisms $\varphi: S^{1}(\simeq M(\infty)) \rightarrow$ $S^{1}\left(\simeq \mathbb{H}^{2}(\infty)\right)$ as prescribed boundary data. This is a converse of the result proved in Choi and Treibergs [7, 8].

Finally, we would like to mention some other related results for harmonic maps. Avilés, Choi, and Micallef [6] and the author [2] proved independently that the asymptotic Dirichlet problem from a strictly negatively curved Hadamard manifold $M$ to a Hadamard manifold $N$ can be solved uniquely for each $C^{0}$ boundary data from $M(\infty)$, the boundary at infinity of $M$, into $N$. On the other hand, nonexistence results for harmonic maps from Euclidean $m$-space $\mathbb{R}^{m}$ to a Hadamard manifold of negative sectional curvature bounded away from zero can be seen in Tachikawa and the author [4, 19].

Acknowledgment. I would like to express my sincere thanks to Professor S. Nishikawa for his continuous encouragement and valuable suggestions and to the referee for kind advice.

\section{Preliminaries AND Statement of MAin Results}

Let $M$ be a Hadamard $n$-manifold. The boundary at infinity or the Eberlein$O$ 'Neill boundary $M(\infty)$ of $M$ is defined to be the set of all asymptotic classes of geodesic rays in $M$; two rays $\gamma_{1}$ and $\gamma_{2}$ are asymptotic if $\operatorname{dist}_{M}\left(\gamma_{1}(t), \gamma_{2}(t)\right)$ is bounded for all $t \geq 0$. One can define on $\bar{M}:=M \cup M(\infty)$ a natural topology, the cone topology of $\bar{M}$, with respect to which the triple $(\bar{M}, M, M(\infty))$ is identified topologically with the Euclidean triple $\left(\bar{B}^{n}, B^{n}, S^{n-1}\right)$, where $B^{n}=$ $\left\{x \in \mathbb{R}^{n+1} ;|x|<1\right\}$ and $S^{n-1}=\partial B^{n}$ (see [9] for details). Note that there exists no natural (i.e., independent of each pole $o \in M$ ) smooth structure on $M(\infty)$ except when $M=\mathbb{H}^{n}$ (see [5]).

The Poincaré model $D$ of the hyperbolic plane $\mathbb{H}^{2}$ is by definition the unit disk $B$ in the complex plane $\mathbb{C}$ equipped with the metric

$$
d s_{D}^{2}=\frac{4 d z d \bar{z}}{\left(1-|z|^{2}\right)^{2}}=\frac{4\left(\left(d x^{1}\right)^{2}+\left(d x^{2}\right)^{2}\right)}{\left(1-\left(x^{1}\right)^{2}-\left(x^{2}\right)^{2}\right)^{2}},
$$

where $z=x^{1}+i x^{2}$ is the canonical global coordinate of $\mathbb{C}$. Throughout this paper, we always denote by $z=x^{1}+i x^{2}$ the global coordinate on $D$ as well as $\bar{B}$ (the closure of $B$ in $\mathbb{C}$ ), and regard $D$ as a Riemann surface by $z$. Also, we often regard $\bar{B}$ as a Riemannian 2-manifold with boundary 
equipped with flat Euclidean metric. It is easy to see that $D$ is a Hadamard 2manifold of constant negative curvature -1 . The cone topology is defined also on $\bar{D}:=D \cup D(\infty)$, and the triple $(\bar{D}, D, D(\infty))$ is identified topologically with the Euclidean triple $\left(\bar{B}, B, S^{1}\right)$. We may regard $D(\infty)$ as a $C^{\infty}$ Riemannian 1-manifold by identifying $D(\infty) \simeq S^{1}$.

Let $\varphi$ be a continuous map of $S^{1}$ to itself. We say that $\varphi$ is conformal if $\varphi$ is the restriction $\left.f\right|_{S^{1}}$ of a holomorphic or antiholomorphic map $f$ of $\bar{B}$ onto itself. By $\mathscr{D}(D), \mathscr{H}(\bar{D}), \mathscr{D}(D, B)$ and $\mathscr{H}(\bar{B}, \bar{D})$ we denote respectively the set of all diffeomorphisms of $D$, the set of all homeomorphisms of $\bar{D}$, the set of all diffeomorphisms of $B$ onto $D$, and the set of all homeomorphisms of $\bar{B}$ onto $\bar{D}$.

Let $\Sigma_{1}=\left(\Sigma_{1}, g\right)$ and $\Sigma_{2}=\left(\Sigma_{2}, h\right)$ be oriented Riemannian 2-manifolds. Let $\left(y^{1}, y^{2}\right)$ and $\left(u^{1}, u^{2}\right)$ denote isothermal coordinates on $\Sigma_{1}$ and $\Sigma_{2}$ respectively, by which $g$ and $h$ are expressed locally as

$$
\begin{aligned}
& g=\sigma(w)^{2}\left(\left(d y^{1}\right)^{2}+\left(d y^{2}\right)^{2}\right)=\sigma^{2} d w d \bar{w}, \quad \sigma>0, \\
& h=\rho(u)^{2}\left(\left(d u^{1}\right)^{2}+\left(d u^{2}\right)^{2}\right)=\rho^{2} d u d \bar{u}, \quad \rho>0,
\end{aligned}
$$

where $w=y^{1}+i y^{2}$ and $u=u^{1}+i u^{2}$. A $C^{2}$ map $u: \Sigma_{1} \rightarrow \Sigma_{2}$ is a harmonic map if $u(w)$, the representation of $u$ in these coordinates, satisfies the following system of equations:

$$
\tau(u)^{j}:=\sigma^{-2} \sum_{\alpha=1}^{2}\left[\frac{\partial^{2} u^{j}}{\left(\partial y^{\alpha}\right)^{2}}+\Gamma_{k l}^{j}(u) \frac{\partial u^{k}}{\partial y^{\alpha}} \frac{\partial u^{l}}{\partial y^{\alpha}}\right]=0 \text { for } j=1,2,
$$

where $\Gamma_{k l}^{j}$ denote the Christoffel symbols of the Levi-Civita connection of $h$. The system (2.2) is equivalent to the equation

$$
\sigma^{-2}\left(\frac{\partial^{2} u}{\partial w \partial \bar{w}}+\frac{2}{\rho} \frac{\partial \rho}{\partial u} \frac{\partial u}{\partial w} \frac{\partial u}{\partial \bar{w}}\right)=0
$$

where

$$
\frac{\partial u}{\partial w}=\frac{1}{2}\left(\frac{\partial u}{\partial y^{1}}-i \frac{\partial u}{\partial y^{2}}\right) \text { and } \frac{\partial u}{\partial \bar{w}}=\frac{1}{2}\left(\frac{\partial u}{\partial y^{1}}+i \frac{\partial u}{\partial y^{2}}\right) .
$$

It is immediate from (2.3) that the harmonicity of $u$ does not depend on the choice of compatible metric of $\Sigma_{1}$, but depends only on its conformal structure, and $u$ is harmonic if $u$ is holomorphic or antiholomorphic.

Finally, we note that it follows from (2.1) and (2.3) that a $C^{2}$ map $u: D \rightarrow D$ is harmonic if and only if $u$ satisfies the equation

$$
\frac{\partial^{2} u}{\partial z \partial \bar{z}}+\frac{2 \bar{u}}{1-|u|^{2}} \frac{\partial u}{\partial z} \frac{\partial u}{\partial \bar{z}}=0 .
$$

With these understood, our main results can be stated as follows.

Theorem 1. Let $\varphi: D(\infty) \rightarrow D(\infty)$ be a $C^{4}$ immersion. Then there exists a harmonic map $u \in C^{\infty}(D, D) \cap C^{0}(\bar{D}, \bar{D})$ such that $\left.u\right|_{D(\infty)}=\varphi$. Moreover $u$ is holomorphic or antiholomorphic if and only if $\varphi$ is conformal.

Theorem 2. Let $\varphi$ be a $C^{4}$ diffeomorphism of $D(\infty)$. Then there exists a harmonic diffeomorphism $u \in \mathscr{D}(D) \cap \mathscr{H}(\bar{D})$ such that $\left.u\right|_{D(\infty)}=\varphi$. Moreover $u$ is conformal if and only if $\varphi$ is conformal. 
Remark 1. If we choose, in Theorem 2, a nonconformal $\varphi$ in particular, then we can obtain a nonconformal harmonic diffeomorphism $u$ of $D$.

\section{Proofs of Theorems 1 and 2}

We first note the following lemma, which is an easy consequence of (2.3).

Lemma 1 (cf. [13]). Let $\Sigma_{1}, \Sigma_{2}$, and $\Sigma_{3}$ be oriented Riemannian 2-manifolds. Suppose that $u: \Sigma_{2} \rightarrow \Sigma_{3}$ is a harmonic map. If $v: \Sigma_{1} \rightarrow \Sigma_{2}$ is a conformal map, then the composition $u \circ v: \Sigma_{1} \rightarrow \Sigma_{3}$ is also a harmonic map.

On account of Lemma 1, we can reduce the proofs of Theorems 1 and 2 to the following.

Theorem 1'. Let $\varphi: S^{1} \rightarrow D(\infty)$ be a $C^{4}$ immersion. Then there exists a harmonic map $u \in C^{\infty}(B, D) \cap C^{0}(\bar{B}, \bar{D})$ such that $\left.u\right|_{S^{1}}=\varphi$. Moreover $u$ is holomorphic or antiholomorphic if and only if $\varphi$ is conformal.

Theorem $2^{\prime}$. Let $\varphi$ be a $C^{4}$ diffeomorphism of $S^{1}$ onto $D(\infty)$. Then there exists a harmonic diffeomorphism $u \in \mathscr{D}(B, D) \cap \mathscr{H}(\bar{B}, \bar{D})$ such that $\left.u\right|_{S^{1}}=\varphi$. Moreover $u$ is conformal if and only if $\varphi$ is conformal.

Let $\varphi: S^{1} \rightarrow S^{1}(\simeq D(\infty))$ be the $C^{4}$ immersion in Theorem $1^{\prime}$. For each $\rho(0<\rho<1)$ let $u_{\rho}: B \rightarrow B_{\rho}:=\left\{z \in D ; \operatorname{dist}_{D}(0, z)<\log [(1+\rho) /(1-\rho)]\right\}$ be a harmonic map with $\left.u_{\rho}\right|_{S^{1}}=\rho \cdot \varphi$ (cf. [13]). Associated with $\varphi$, we will first construct a barrier map (or an approximation) $\Phi: \bar{B} \rightarrow \bar{B}(\simeq \bar{D})$ at the boundary $S^{1} ; \Phi$ needs to satisfy the boundary conditions $\left.\Phi\right|_{S^{1}}=\varphi$ and the inequality (3.11) in Lemma 4 below. Then from these properties and a priori gradient estimates for harmonic maps in [14], we will prove that a subsequence $\left\{u_{\rho_{j}}\right\}_{j \in \mathbb{N}}$ of $\left\{u_{\rho}\right\}_{0<\rho<1}$ converges to a harmonic map $u: B \rightarrow D$ such that $\left.u\right|_{S^{1}}=\varphi$.

Now, we shall prove Theorem $1^{\prime}$ by a series of lemmas. Note that, without loss of generality, we may assume $\operatorname{deg}(\varphi)>0$ throughout this section. Let $\Theta: \mathbb{R} \rightarrow \mathbb{R}$ denote a $C^{4}$ diffeomorphism which satisfies $\varphi\left(e^{i \theta}\right)=e^{i \boldsymbol{\theta}(\theta)}$.

Lemma 2. Let $\mathscr{V}_{1}$ be a neighborhood of $S^{1}$ in $\mathbb{C}$. Define a $C^{2}$ map $\tilde{\varphi}=$ $\tilde{\varphi}^{1}+i \tilde{\varphi}^{2}: \mathscr{V}_{1} \rightarrow \mathbb{C} b y$

$$
\tilde{\varphi}(z)=e^{i \boldsymbol{\Theta}}+e^{i \boldsymbol{\Theta}} \cdot \boldsymbol{\Theta}^{\prime} \cdot(r-1)+\frac{1}{2} e^{i \boldsymbol{\Theta}}\left[-\boldsymbol{\Theta}^{\prime}+\left(\boldsymbol{\Theta}^{\prime}\right)^{2}-i \boldsymbol{\Theta}^{\prime \prime}\right](r-1)^{2}
$$

for all $z=r e^{i \theta} \in \mathscr{V}_{1}$, where prime denotes differentiation with respect to $\theta$. Then the following hold.

$$
\begin{gathered}
\tilde{\varphi}=\varphi \quad \text { on } S^{1}, \\
1-|\tilde{\varphi}|^{2}=(1-r) \cdot \Theta^{\prime}+(1-r)^{2} f_{0} \quad \text { on } \mathscr{V}_{1}, \\
\left|\frac{\partial \tilde{\varphi}}{\partial \bar{z}}\right|=(1-r)^{2} f_{1} \quad \text { on } \mathscr{V}_{1}, \\
\left|\frac{\partial^{2} \tilde{\varphi}}{\partial z \partial \bar{z}}\right|=(1-r) f_{2} \quad \text { on } \mathscr{V}_{1},
\end{gathered}
$$


where $f_{j}(0 \leq j \leq 2)$ are bounded $C^{0}$ functions (depending only on the boundary data $\varphi)$ on $\mathscr{V}_{1}$. Moreover, on a neighborhood $\mathscr{V}_{2}\left(\subset \mathscr{V}_{1}\right)$ of $S^{1},\left.\tilde{\varphi}\right|_{\mathscr{V}_{2}}: \mathscr{V}_{2} \rightarrow \mathbb{C}$ is a $C^{2}$ immersion.

Proof. (3.2) and (3.3) follow directly from (3.1). Remark that $\Theta^{\prime}$ has a positive infimum, since $\varphi: S^{1} \rightarrow S^{1}$ is a $C^{4}$ immersion. Using the formulas

$$
\begin{aligned}
\frac{\partial}{\partial z} & =\frac{1}{2} e^{-i \theta}\left(\frac{\partial}{\partial r}-\frac{i}{r} \frac{\partial}{\partial \theta}\right), \\
\frac{\partial}{\partial \bar{z}} & =\frac{1}{2} e^{i \theta}\left(\frac{\partial}{\partial r}+\frac{i}{r} \frac{\partial}{\partial \theta}\right), \\
\frac{\partial^{2}}{\partial z \partial \bar{z}} & =\frac{1}{4}\left[\left(\frac{\partial}{\partial r}\right)^{2}+\frac{1}{r} \frac{\partial}{\partial r}+\frac{1}{r^{2}}\left(\frac{\partial}{\partial \theta}\right)^{2}\right],
\end{aligned}
$$

we have on $S^{1}$

$$
\left|\frac{\partial \tilde{\varphi}}{\partial \bar{z}}\right|=0, \quad\left|\frac{\partial}{\partial r}\left(\frac{\partial \tilde{\varphi}}{\partial \bar{z}}\right)\right|=0, \quad\left|\frac{\partial^{2} \tilde{\varphi}}{\partial z \partial \bar{z}}\right|=0, \quad J(\tilde{\varphi})=\left(\mathbf{\Theta}^{\prime}\right)^{2},
$$

from which (3.4), (3.5) and the last assertion are immediate, where $J(\tilde{\varphi})$ stands for the Jacobian $|\partial \tilde{\varphi} / \partial z|^{2}-|\partial \tilde{\varphi} / \partial \bar{z}|^{2}$ of $\tilde{\varphi}$.

For $\rho(0<\rho<1)$ let $B_{\rho}$ denote either $\{z \in B ;|z|<\rho\}$ or $\{z \in$ $\left.D ; \operatorname{dist}_{D}(0, z)<\log [(1+\rho) /(1-\rho)]\right\}$. From Lemma 2, we see that there exist constants $\rho_{1}, \rho_{2}\left(0<\rho_{1}, \rho_{2}<1\right)$ and a $C^{2}$ map $\Phi:=\Phi^{1}+i \Phi^{2}: \bar{B} \rightarrow \bar{B}$ such that

$$
\bar{B} \backslash B_{\rho_{1}} \subset \mathscr{V}_{2},\left.\quad \Phi\right|_{\bar{B} \backslash B_{\rho_{1}}}=\tilde{\varphi}, \quad \Phi\left(\bar{B}_{\rho_{1}}\right) \subset \bar{B}_{\rho_{2}} .
$$

Let $\Phi_{\rho}$ denote the $C^{2}$ map $\rho \cdot \Phi: \bar{B} \rightarrow \bar{B}_{\rho}$, and $\varphi_{\rho}$, the $C^{4}$ immersion $\rho \cdot \varphi: S^{1} \rightarrow \partial \bar{B}_{\rho}$. By the existence and uniqueness theorem of harmonic maps $[10,11,12]$, there exists a unique harmonic map $u_{\rho} \in C^{\infty}\left(B, B_{\rho}\right) \cap C^{3, \alpha}\left(\bar{B}, \bar{B}_{\rho}\right)$ (for any $\alpha \in(0,1)$ ) such that $\left.u_{\rho}\right|_{S^{1}}=\varphi_{\rho}$. Let $Z_{\rho}=\left\{z \in \bar{B} ; u_{\rho}(z)=\Phi_{\rho}(z)\right\}$, the coincidence set of $u_{\rho}$ and $\Phi_{\rho}$. We shall next prove a priori growth estimates of $u_{\rho}$.

Lemma 3. For each $\rho(0<\rho<1)$

$$
\Delta\left\{\operatorname{dist}_{D}\left(u_{\rho}(z), \Phi_{\rho}(z)\right)\right\} \geq-C_{1}
$$

for all $z \in B \backslash Z_{\rho}$, where $\Delta$ stands for the standard Laplacian $4\left(\partial^{2} / \partial z \partial \bar{z}\right)$ on $\mathbb{C}\left(\simeq \mathbb{R}^{2}\right)$ and $C_{1}$ is a positive constant depending only on the boundary data $\varphi$.

Proof. Define $\Lambda: D \times D \rightarrow \mathbb{R}$ by $\Lambda\left(z_{1}, z_{2}\right)=\operatorname{dist}_{D}\left(z_{1}, z_{2}\right)$ for $z_{1}, z_{2} \in D$. Using chain rule, we compute on $B \backslash Z_{\rho}$

$$
\begin{aligned}
& \Delta\left[\operatorname{dist}_{D}\left(u_{\rho}(z), \Phi_{\rho}(z)\right)\right] \\
& =\sum_{\alpha=1}^{2}(\operatorname{Hess}(\Lambda))\left(\left(u_{\rho}\right)_{*}\left(\frac{\partial}{\partial x^{\alpha}}\right) \oplus\left(\Phi_{\rho}\right)_{*}\left(\frac{\partial}{\partial x^{\alpha}}\right),\right. \\
& \left.\quad\left(u_{\rho}\right)_{*}\left(\frac{\partial}{\partial x^{\alpha}}\right) \oplus\left(\Phi_{\rho}\right)_{*}\left(\frac{\partial}{\partial x^{\alpha}}\right)\right) \\
& \quad+(d \Lambda)\left(\tau\left(u_{\rho}\right) \oplus \tau\left(\Phi_{\rho}\right)\right) .
\end{aligned}
$$


It is not hard to see by a similar argument in the proof of Lemma 3 in [12], that on $B \backslash Z_{\rho}$

$$
(\operatorname{Hess}(\Lambda))\left(\left(u_{\rho}\right)_{*}\left(\frac{\partial}{\partial x^{\alpha}}\right) \oplus\left(\Phi_{\rho}\right)_{*}\left(\frac{\partial}{\partial x^{\alpha}}\right),\left(u_{\rho}\right)_{*}\left(\frac{\partial}{\partial x^{\alpha}}\right) \oplus\left(\Phi_{\rho}\right)_{*}\left(\frac{\partial}{\partial x^{\alpha}}\right)\right) \geq 0
$$

for $\alpha=1,2$. Since $u_{\rho}$ is harmonic (i.e., $\tau\left(u_{\rho}\right)=0$ ), we then have for $z \in B \backslash Z_{\rho}$

$$
\begin{aligned}
\Delta\left[\operatorname{dist}_{D}\left(u_{\rho}(z), \Phi_{\rho}(z)\right)\right] & \\
\geq & \left((d \Lambda)\left(u_{\rho}(z), \Phi_{\rho}(z)\right)\right)\left(0 \oplus \sum_{j=1}^{2} \tau\left(\Phi_{\rho}\right)^{j} \frac{\partial}{\partial x^{j}}\right) \\
& \geq-\left|\sum_{j=1}^{2} \tau\left(\Phi_{\rho}\right)^{j} \frac{\partial}{\partial x^{j}}\right|_{D} \geq-\frac{2}{1-\left|\Phi_{\rho}\right|^{2}}\left|\tau\left(\Phi_{\rho}\right)\right|,
\end{aligned}
$$

where $|\cdot|_{D}$ is the norm with respect to $d s_{D}^{2}$ and $|\cdot|$ the Euclidean norm.

$\tau\left(\Phi_{\rho}\right)$ is given explicitly as

$$
\begin{aligned}
\left|\tau\left(\Phi_{\rho}\right)\right| & =\left|\frac{\partial^{2} \Phi_{\rho}}{\partial z \partial \bar{z}}+\frac{2 \bar{\Phi}_{\rho}}{1-\left|\Phi_{\rho}\right|^{2}} \frac{\partial \Phi_{\rho}}{\partial z} \frac{\partial \Phi_{\rho}}{\partial \bar{z}}\right| \\
& \leq\left|\frac{\partial^{2} \Phi_{\rho}}{\partial z \partial \bar{z}}\right|+\frac{2}{1-\left|\Phi_{\rho}\right|^{2}}\left|\bar{\Phi}_{\rho}\right| \cdot\left|\frac{\partial \Phi_{\rho}}{\partial z}\right| \cdot\left|\frac{\partial \Phi_{\rho}}{\partial \bar{z}}\right| \\
& \leq \rho\left|\frac{\partial^{2} \Phi}{\partial z \partial \bar{z}}\right|+\frac{2 \rho^{3}}{1-\rho^{2}|\Phi|^{2}}|\bar{\Phi}| \cdot\left|\frac{\partial \Phi}{\partial z}\right| \cdot\left|\frac{\partial \Phi}{\partial \bar{z}}\right| .
\end{aligned}
$$

From (3.4) and (3.5) we then obtain

$$
\left|\tau\left(\Phi_{\rho}\right)\right| \leq C\left[(1-r)+\frac{(1-r)^{2}}{1-|\Phi|^{2}}\right]
$$

and hence

$$
\frac{2}{1-\left|\Phi_{\rho}\right|^{2}}\left|\tau\left(\Phi_{\rho}\right)\right| \leq C\left[\frac{1-r}{1-|\Phi|^{2}}+\left(\frac{1-r}{1-|\Phi|^{2}}\right)^{2}\right] .
$$

From (3.3) we also obtain

$$
1-|\Phi|^{2}=(1-r) f_{3},
$$

$f_{3}$ being a bounded positive $C^{0}$ function on $\bar{B}$. It now follows from (3.8) and (3.9) that on $\bar{B}$

$$
\frac{2}{1-\left|\Phi_{\rho}\right|^{2}}\left|\tau\left(\Phi_{\rho}\right)\right| \leq C_{2},
$$

where $C_{2}$ is a constant depending only on $\varphi$. Hence we obtain the estimate (3.7).

Lemma 4. For each $\rho(0<\rho<1)$

$$
\operatorname{dist}_{D}\left(u_{\rho}(z), \Phi_{\rho}(z)\right) \leq \frac{1}{4} C_{1} \text { for all } z \in \bar{B} .
$$


Proof. From (3.7) we have

$$
\Delta\left[\operatorname{dist}_{D}\left(u_{\rho}(z), \Phi_{\rho}(z)\right)+\frac{1}{4} C_{1}|z|^{2}\right] \geq 0 \text { for all } z \in B \backslash Z_{\rho} .
$$

Noting that $\Phi_{\rho}=\varphi_{\rho}=u_{\rho}$ on $S^{1}$, it follows from definition of $Z_{\rho}$ and the maximum principle that

$$
\operatorname{dist}_{D}\left(u_{\rho}(z), \Phi_{\rho}(z)\right)+\frac{1}{4} C_{1}|z|^{2} \leq \frac{1}{4} C_{1} \quad \text { for all } z \in \bar{B}
$$

from which (3.11) follows.

Lemma 5. Represent $\Phi$ and $u_{\rho}$ as $\Phi(z)=L(z) e^{i \cdot \arg (\Phi(z))}$ and $u_{\rho}(z)=$ $R_{\rho}(z) e^{i \cdot \arg \left(u_{\rho}(z)\right)}$ for $z \in \bar{B}$, where $L(z)=|\Phi(z)|$ and $R_{\rho}(z)=\left|u_{\rho}(z)\right|$. Then for all $z \in \bar{B}$ and $\rho(0<\rho<1)$ we have

$$
e^{-C_{1} / 4}(1-\rho \cdot L(z)) \leq 1-R_{\rho}(z) \leq e^{C_{1} / 4}(1-\rho \cdot L(z)),
$$

$$
\begin{aligned}
& \cos \left[\arg \left(u_{\rho}(z)\right)-\arg (\Phi(z))\right] \\
& \quad \geq\left[\cosh ^{2}\left(\log \frac{1+K_{\rho}(z)}{1-K_{\rho}(z)}\right)-\cosh \left(\frac{1}{4} C_{1}\right)\right] / \sinh ^{2}\left(\log \frac{1+K_{\rho}(z)}{1-K_{\rho}(z)}\right),
\end{aligned}
$$

where $K_{\rho}(z)=\min \left\{\rho \cdot L(z), R_{\rho}(z)\right\}$.

Proof. It follows from (3.11) that

$$
\begin{aligned}
\frac{1}{4} C_{1} & \geq \operatorname{dist}_{D}\left(u_{\rho}, \Phi_{\rho}\right) \geq\left|\operatorname{dist}_{D}\left(0, u_{\rho}\right)-\operatorname{dist}_{D}\left(0, \Phi_{\rho}\right)\right| \\
& \geq\left|\log \frac{1+R_{\rho}}{1-R_{\rho}}-\log \frac{1+\rho \cdot L}{1-\rho \cdot L}\right|
\end{aligned}
$$

which implies (3.12). On the other hand, by the law of cosines of geodesic triangles in $D$ we get

$$
\begin{aligned}
\cos [\arg & \left.\left(u_{\rho}\right)-\arg (\Phi)\right] \\
= & {\left[\cosh \left(\operatorname{dist}_{D}\left(0, u_{\rho}\right)\right) \cosh \left(\operatorname{dist}_{D}\left(0, \Phi_{\rho}\right)\right)-\cosh \left(\operatorname{dist}_{D}\left(u_{\rho}, \Phi_{\rho}\right)\right)\right] } \\
& \times\left[\sinh \left(\operatorname{dist}_{D}\left(0, u_{\rho}\right)\right) \sinh \left(\operatorname{dist}_{D}\left(0, \Phi_{\rho}\right)\right)\right]^{-1} \\
\geq & {\left[\cosh ^{2}\left(\log \frac{1+K_{\rho}}{1-K_{\rho}}\right)-\cosh \left(\operatorname{dist}_{D}\left(u_{\rho}, \Phi_{\rho}\right)\right)\right] / \sinh ^{2}\left(\log \frac{1+K_{\rho}}{1-K_{\rho}}\right), }
\end{aligned}
$$

which, together with (3.11), then implies (3.13).

Lemma 6. For each $k(0<k<1)$, there exists a constant $l=l(k, \varphi) \quad(0<$ $l<1)$ such that

$$
u_{\rho}\left(\bar{B}_{k}\right) \subset \bar{B}_{l} \text { for all } \rho(0<\rho<1) .
$$

Proof. First it follows from (3.3) and (3.6) that there exists a constant $l_{0} \quad(0<$ $\left.l_{0}<1\right)$ such that

$$
\Phi_{\rho}\left(\bar{B}_{k}\right) \subset \Phi\left(\bar{B}_{k}\right) \subset \bar{B}_{l_{0}} \text { for all } \rho \text {. }
$$

Using (3.12) in (3.15) then yields

$$
u_{\rho}\left(\bar{B}_{k}\right) \subset \bar{B}_{l} \text { for all } \rho,
$$

where $l=1-e^{-C_{1} / 4}\left(1-l_{0}\right)$.

For each $k \quad(0<k<1)$, from (3.14) we have

$$
u_{\rho}\left(\bar{B}_{(1+k) / 2}\right) \subset \bar{B}_{l_{1}} \text { for all } \rho(0<\rho<1) \text {, }
$$


where $l_{1}=l_{1}(k, \varphi) \quad\left(0<l_{1}<1\right)$. Then it follows from a priori gradient estimates for harmonic maps in $[13,14]$ that for all $\rho(0<\rho<1)$

$$
\sup _{z \in B_{k}}\left\|d u_{\rho}(z)\right\| \leq C \sup _{z_{1} \in B_{k}} \frac{\operatorname{dist}_{D}\left(u_{\rho}\left(z_{1}\right), u_{\rho}\left(z_{2}\right)\right)}{(1-k) / 2}, \quad z_{2} \in B_{(1-k) / 2}\left(z_{1}\right),
$$

where $C=C\left(k, l_{1}\right)$ and $B_{(1-k) / 2}\left(z_{1}\right)=\left\{z \in B ;\left|z-z_{1}\right|<\frac{1}{2}(1-k)\right\}$. In consequence, it follows from (3.16) that

$$
\sup _{z \in B_{k}}\left\|d u_{\rho}(z)\right\| \leq C\left(4 \log \frac{1+l_{1}}{1-l_{1}}\right)(1-k)^{-1}
$$

for all $\rho(0<\rho<1)$.

Let $\left\|u_{\rho}\right\|_{C^{2, \alpha}\left(B_{k}\right)}$ denote the $C^{2, \alpha}$-Hölder norm of $u_{\rho} \in C^{\infty}(B, D)$ in $B_{k}$. Then it also follows from (3.17) and a priori $C^{2, \alpha}$-estimates in $[13,14]$ that for each $\alpha \quad(0<\alpha<1)$

$$
\left\|u_{\rho}\right\|_{C^{2, \alpha}\left(B_{k}\right)} \leq C_{3}
$$

for all $\rho(0<\rho<1)$, where $C_{3}=C_{3}\left(\alpha, k, l_{1}\right)$.

Once these are established, it follows from (3.14), (3.18) and the AscoliArzelá theorem that there exist a subsequence $\left\{u_{\rho_{j}}\right\}_{j \in \mathbb{N}}$ of $\left\{u_{\rho}\right\}_{0<\rho<1}$ and a harmonic map $u \in C^{2}(B, D)$ such that on every compact subset of $B, u_{\rho_{j}}$ converges to $u$ in the $C^{2}$ topology. The harmonicity of $u$ then implies $u \in$ $C^{\infty}(B, D)$.

Put $u(z)=R(z) e^{i \cdot \arg (u(z))}$ for $z \in B$, where $R(z)=|u(z)|$. From (3.11)(3.13) the following then holds for $u$.

Lemma 7. For all $z \in B$ we have

$$
\begin{gathered}
\operatorname{dist}_{D}(u(z), \Phi(z)) \leq \frac{1}{4} C_{1}, \\
e^{-C_{1} / 4}(1-L(z)) \leq 1-R(z) \leq e^{C_{1} / 4}(1-L(z)),
\end{gathered}
$$

$$
\begin{aligned}
& \cos [\arg (u(z))-\arg (\Phi(z))] \\
& \quad \geq\left[\cosh ^{2}\left(\log \frac{1+K(z)}{1-K(z)}\right)-\cosh \left(\frac{1}{4} C_{1}\right)\right] / \sinh ^{2}\left(\log \frac{1+K(z)}{1-K(z)}\right),
\end{aligned}
$$

where $K(z)=\min \{L(z), R(z)\}$.

Lemma 8. $u$ extends to a continuous map of $\bar{B}$ onto $\bar{D}$ satisfying $\left.u\right|_{S^{1}}=\varphi$.

Proof. Take a point $z_{0} \in S^{1}$ and a neighborhood $T(\subset \bar{D})$ of $\varphi\left(z_{0}\right) \in D(\infty)$. Let $W \in W\left(\varphi\left(z_{0}\right), \delta\right)$ be a neighborhood $\left\{z \in \bar{D} ;\left|z-\varphi\left(z_{0}\right)\right|<\delta\right\}$ satisfying $W \subset T$ and

$$
\operatorname{dist}_{D}\left(z_{1}, z_{2}\right) \geq \frac{1}{2} C_{1}
$$

for $z_{1} \in W \cap D$ and $z_{2} \in \partial T \cap D$. Then, from (3.2) and (3.6), there exists a positive constant $\varepsilon=\varepsilon(\varphi, \delta)$ such that

$$
\Phi\left(\mathscr{V}\left(z_{0}, \varepsilon\right)\right) \subset W
$$

where $\mathscr{V}\left(z_{0}, \varepsilon\right)=\left\{z \in B ;\left|z-z_{0}\right|<\varepsilon\right\}$. It then follows from (3.19), (3.22), and (3.23) that $u\left(\mathscr{V}\left(z_{0}, \varepsilon\right)\right) \subset T$. This implies that $\lim _{z \rightarrow z_{0}} u(z)=\varphi\left(z_{0}\right)$. Since $z_{0} \in S^{1}$ is arbitrary, $\left.u\right|_{S^{1}}=\varphi$. 
Lemma 9. $u$ is holomorphic if and only if $\varphi$ is conformal.

Proof. We first assume that $\varphi$ is conformal. Since $\operatorname{deg}(\varphi)>0$ by assumption, there exists, by the argument principle, a holomorphic map $f$ of $\bar{B}$ onto $\bar{B}$ $(\simeq \bar{D})$ such that $\left.f\right|_{S^{1}}=\varphi$. It then follows from the uniqueness of $u_{\rho}$ that $u_{\rho}=\rho \cdot f$ for all $\rho(0<\rho<1)$. Hence $u=f$.

Next, we assume that $u$ is holomorphic. Since $\left.u\right|_{S^{1}}=\varphi, u$ has only finitely many zeros $z_{1}, \ldots, z_{n}$ in $B$. By the Poisson-Jensen formula (cf. [1]), we then obtain

$$
\log \left|u(z) \prod_{j=1}^{n}\left(\frac{1-\bar{z}_{j} z}{z-z_{j}}\right)\right|=\frac{1}{2 \pi} \int_{0}^{2 \pi} \operatorname{Re}\left(\frac{e^{i \theta}+z}{e^{i \theta}-z}\right) \log \left|u\left(e^{i \theta}\right)\right| d \theta,
$$

which implies

$$
u(z)=e^{i \alpha} \prod_{j=1}^{n}\left(\frac{z-z_{j}}{1-\bar{z}_{j} z}\right) \quad \text { for } z \in B,
$$

where $\alpha$ is a real constant, since $\left.u\right|_{S^{1}}=\varphi$. It follows from (3.24) that $u$ extends to a holomorphic map of $\bar{B}$ onto $\bar{B}(\simeq D)$ with $\left.u\right|_{S^{1}}=\varphi$. Hence $\varphi$ is conformal. We note that $\operatorname{deg}(\varphi)=n$. This completes the proof of Theorem $1^{\prime}$.

Remark 2. When $\varphi$ is not conformal, it follows from Lemma 12.2 in [13] and Lemma 9 that the zeros of $|\partial u / \partial z|$ or $|\partial u / \partial \bar{z}|$ are isolated in $B$.

Proof of Theorem $2^{\prime}$. Let $u$ be a harmonic map constructed in the proof of Theorem $1^{\prime}$. To prove Theorem $2^{\prime}$, it suffices to show $u \in \mathscr{D}(B, D)$ when $\operatorname{deg}(\varphi)= \pm 1$. We may assume, without loss of generality, that $\operatorname{deg}(\varphi)=1$.

On account of the existence theorem of harmonic diffeomorphisms [13, 18], we have

$$
u_{\rho} \in \mathscr{D}\left(B, B_{\rho}\right) \cap C^{3, \alpha}\left(\bar{B}, \bar{B}_{\rho}\right) \text { for all } \rho(0<\rho<1) \text {, }
$$

where $0<\alpha<1$. Combining (3.3) and (3.6) with (3.12), it is verified that for each $k \quad\left(0<\rho_{1}<k<1\right)$, there exist constants $l, l^{\prime}$ and $\rho_{3} \quad\left(0<\rho_{3}<1\right.$, $\left.0<l<l^{\prime}<1\right)$ such that

$$
\bar{B}_{l} \subset u_{\rho}\left(\bar{B}_{(1+k) / 2}\right) \subset \bar{B}_{l^{\prime}} \text { for all } \rho \geq \rho_{3} .
$$

It then follows from (3.25) together with Theorem 7.1 in [13] that

$$
\left(J\left(u_{\rho}\right)\right)(z) \geq\left(\left(1-\left|u_{\rho}(z)\right|^{2}\right) / 2\right)^{2} \delta^{-1}>0
$$

for all $\rho \geq \rho_{3}$ and $z \in B_{k}$, where $\delta=\delta\left(k, l, l^{\prime}\right)>0$. Applying (3.26) to the subsequence $\left\{u_{\rho_{j}}\right\}_{j \in \mathbb{N}}$ and letting $\rho_{j} \rightarrow 1$, we know that $u$ satisfies

$$
(J(u))(z) \geq\left(\left(1-|u(z)|^{2}\right) / 2\right)^{2} \delta^{-1}>0 \text { for all } z \in B_{k},
$$

and then from (3.3) and (3.20)

$$
(J(u))(z)>0 \text { for all } z \in B,
$$

which implies that $u$ is a local diffeomorphism of $B$ to $D$. Hence $u \in$ $\mathscr{D}(B, D)$, completing the proof of Theorem $2^{\prime}$. 
Remark 3. Since

$$
J(u)=|\partial u / \partial z|^{2}-|\partial u / \partial \bar{z}|^{2},
$$

it follows from (3.27) and (3.28) that

$$
|\partial u / \partial z|>0(\text { resp. }|\partial u / \partial \bar{z}|>0) \text { in } B \text {, }
$$

if $\operatorname{deg}(\varphi)=1(\operatorname{resp} . \operatorname{deg}(\varphi)=-1)$.

4. THE BOUNDARY BeHAVIOR OF $|d u|$ AND $J(u)$

In this section, we shall investigate the boundary behavior of $|d u|$ and $|J(u)|$, which will be of use in $\S 5$.

Proposition 1. Let $u$ be a harmonic map constructed in Theorem 1'. Regard $u$ as a map $u \in C^{\infty}(B, B) \cap C^{0}(\bar{B}, \bar{B})$. Then $u$ is a Lipschitz map of $\bar{B}$ onto itself, i.e., there exists a positive constant $C_{4}$ depending only on $\varphi$ such that

$$
\left|\sum_{j, \alpha=1}^{2}\left(\frac{\partial u^{j}}{\partial x^{\alpha}}\right)^{2}(z)\right| \leq C_{4} \quad \text { for } z \in B .
$$

Proof. We first regard $u$ and $\Phi$ as maps $u, \Phi \in C^{\infty}(D, D) \cap C^{0}(\bar{D}, \bar{D})$. Let $\sigma$ be a positive constant and fix it. Take a point $z_{0}$ in $D$ satisfying

$$
\log \frac{1+\left|z_{0}\right|}{1-\left|z_{0}\right|} \geq 4 \sigma \text {. }
$$

Let $\widetilde{B}_{\sigma}\left(z_{0}\right)$ denote the open geodesic ball $\left\{z \in D ; \operatorname{dist}_{D}\left(z, z_{0}\right)<\sigma\right\}$.

We shall estimate $\widetilde{B}_{\sigma}\left(z_{0}\right)$ from the outside by a truncated cone. Let $r_{1}=$ $r_{1}\left(\left|z_{0}\right|\right), r_{2}=r_{2}\left(\left|z_{0}\right|\right)$, and $\xi=\xi\left(z_{0}\right) \quad(0<\xi<\pi / 2)$ be constants satisfying

$$
0<r_{1}<\left|z_{0}\right|<r_{2}<1 \text {, }
$$

$$
\begin{aligned}
& \sigma=\log \left(\frac{1-r_{1}}{1-\left|z_{0}\right|} \cdot \frac{1+\left|z_{0}\right|}{1+r_{1}}\right)=\log \left(\frac{1-\left|z_{0}\right|}{1-r_{2}} \cdot \frac{1+r_{2}}{1+\left|z_{0}\right|}\right) . \\
& \cosh (2 \sigma)=\cosh ^{2}\left(\log \frac{1+\left|z_{0}\right|}{1-\left|z_{0}\right|}\right)-\cos \xi \sinh ^{2}\left(\log \frac{1+\left|z_{0}\right|}{1-\left|z_{0}\right|}\right) .
\end{aligned}
$$

Then it is immediate from (4.2)-(4.4) that the truncated cone

$$
\mathscr{T}=\mathscr{T}_{0}\left(z_{0}, r_{1}, r_{2}, \xi\right)=\left\{z \in D ; r_{1}<|z|<r_{2}, \varangle_{0}\left(z, z_{0}\right)<\xi\right\}
$$

satisfies

$$
\widetilde{B}_{\sigma}\left(z_{0}\right) \subset \mathscr{T}
$$

where $\varangle_{0}\left(z, z_{0}\right)$ denotes the angle between the vectors $\overrightarrow{0 z}$ and $\overrightarrow{0 z_{0}}$ in $B$.

Next, we shall estimate $\Phi(\mathscr{T})$ from the outside by a geodesic ball. It follows from (3.3) and (3.6) that there exist a bounded positive $C^{0}$ function $h$ of $\bar{B}$ and a constant $r_{0} \quad\left(0<\rho_{1}<r_{0}<1\right)$ such that

$$
\begin{gathered}
1-|\Phi|=(1-r) h \quad \text { on } \bar{B} \\
|\partial(\arg (\Phi)) / \partial \theta| \leq C_{5} \text { on } \bar{B} \backslash B_{r_{0}},
\end{gathered}
$$


where $C_{5}=C_{5}(\varphi)$. It is an immediate consequence of (4.3) and (4.6) that we have the estimate

$$
\begin{aligned}
\mid \operatorname{dist}_{D} & (\Phi(z), 0)-\operatorname{dist}_{D}\left(\Phi\left(z_{0}\right), 0\right) \mid \\
& =\left|\log \left(\frac{1-|\Phi(z)|}{1-\left|\Phi\left(z_{0}\right)\right|} \cdot \frac{1+\left|\Phi\left(z_{0}\right)\right|}{1+|\Phi(z)|}\right)\right| \\
& \leq\left|\log \frac{1-|z|}{1-\left|z_{0}\right|}\right|+\log \left(2 A_{0}\right) \\
& \leq \sigma+\log \left(2 A_{0}\right) \quad \text { for all } z \in \mathscr{T},
\end{aligned}
$$

where $A_{0}=\sup _{\bar{B}} h / \inf _{\bar{B}} h$. Also, form (4.3), (4.4), and (4.7), there exists a constant $r_{3} \quad\left(r_{0}<r_{3}<1\right)$ such that

$$
\begin{gathered}
0<C_{5} \xi<\pi / 2, \\
0<1-\cos \left(C_{5} \xi\right)<2\left(C_{5}\right)^{2}(1-\cos \xi) \text { for } r_{1}=r_{1}\left(\left|z_{0}\right|\right) \geq r_{3} .
\end{gathered}
$$

Note that if $\left|z_{0}\right| \geq(\sqrt{2}-1) /(\sqrt{2}+1)$, then

$$
\sinh \left(\log \left(\frac{1+\left|z_{0}\right|}{1-\left|z_{0}\right|} t\right)\right) \leq 2 t \sinh \left(\log \frac{1+\left|z_{0}\right|}{1-\left|z_{0}\right|}\right) \quad \text { for } t>0 .
$$

Put now $r_{4}=\max \left\{r_{3},(\sqrt{2}-1) /(\sqrt{2}+1)\right\}$. Then, when $r_{1}=r_{1}\left(\left|z_{0}\right|\right) \geq r_{4}$, it follows from (4.4), (4.6), (4.7), (4.9), and (4.10) that we have for $z \in \mathscr{T}$

$$
\begin{aligned}
\cosh ( & \left.\operatorname{dist}_{D}\left(\Phi\left(z_{0}\right),\left|\Phi\left(z_{0}\right)\right| e^{i \cdot \arg (\Phi(z))}\right)\right) \\
= & \cosh ^{2}\left(\log \frac{1+\left|\Phi\left(z_{0}\right)\right|}{1-\left|\Phi\left(z_{0}\right)\right|}\right) \\
& -\cos \left[\arg (\Phi(z))-\arg \left(\Phi\left(z_{0}\right)\right)\right] \sinh ^{2}\left(\log \frac{1+\left|\Phi\left(z_{0}\right)\right|}{1-\left|\Phi\left(z_{0}\right)\right|}\right) \\
= & 1+\left[1-\cos \left\{\arg (\Phi(z))-\arg \left(\Phi\left(z_{0}\right)\right)\right\}\right] \sinh ^{2}\left(\log \frac{1+\left|\Phi\left(z_{0}\right)\right|}{1-\left|\Phi\left(z_{0}\right)\right|}\right) \\
\leq & 1+\left(1-\cos \left(C_{5} \xi\right)\right) \sinh ^{2}\left(\log \left(\frac{1+\left|z_{0}\right|}{1-\left|z_{0}\right|} \frac{2}{A_{1}}\right)\right) \\
\leq & 1+2\left(C_{5}\right)^{2}(1-\cos \xi)\left(\frac{4}{A_{1}}\right)^{2} \sinh ^{2}\left(\log \frac{1+\left|z_{0}\right|}{1-\left|z_{0}\right|}\right) \\
\leq & C_{6}\left[\cosh \left(\log \frac{1+\left|z_{0}\right|}{1-\left|z_{0}\right|}\right)-\cos \xi \sinh ^{2}\left(\log \frac{1+\left|z_{0}\right|}{1-\left|z_{0}\right|}\right)\right] \\
= & C_{6} \cosh (2 \sigma)
\end{aligned}
$$

and hence

$$
\operatorname{dist}_{D}\left(\Phi\left(z_{0}\right),\left|\Phi\left(z_{0}\right)\right| e^{i \cdot \arg (\Phi(z))}\right) \leq \log \left(2 C_{6} \cosh (2 \sigma)\right),
$$

where $A_{1}=\inf _{\bar{B}} h(\leq 1)$ and $C_{6}=\max \left\{1,32\left(C_{5} / A_{1}\right)^{2}\right\}$. On the other hand, when $r_{1}=r_{1}\left(\left|z_{0}\right|\right)<r_{4}$ or $\log \left[\left(1+\left|z_{0}\right|\right) /\left(1-\left|z_{0}\right|\right)\right]<4 \sigma$, we get from (4.3) and

$$
\Phi(\mathscr{T}) \subset B_{r_{5}}
$$

where $r_{5}=r_{5}\left(\sigma, r_{4}, h\right) \quad\left(0<r_{5}<1\right)$. By putting

$$
\lambda=\max \left\{\sigma+\log \left(2 A_{0}\right)+\log \left(2 C_{6} \cosh (2 \sigma)\right), 2 \log \frac{1+r_{5}}{1-r_{5}}\right\},
$$


it then follows from (4.8), (4.11) and (4.12) that

$$
\Phi(\mathscr{T}) \subset \widetilde{B}_{\lambda}\left(\Phi\left(z_{0}\right)\right) .
$$

We now obtain from (3.19), (4.5), and (4.13) that

$$
u\left(\widetilde{B}_{\sigma}\left(z_{0}\right)\right) \subset \widetilde{B}_{\lambda+C_{1} / 2}\left(u\left(z_{0}\right)\right) .
$$

It is verified from (4.14) and a priori gradient estimates for harmonic maps (Theorem 6.1 in [13]) that

$$
\frac{1-\left|z_{0}\right|^{2}}{1-\left|u\left(z_{0}\right)\right|^{2}}\left|\sum_{j, \alpha=1}^{2}\left(\frac{\partial u^{j}}{\partial x^{\alpha}}\right)^{2}\left(z_{0}\right)\right|^{1 / 2} \leq \frac{C_{7}\left(\lambda+\frac{1}{2} C_{1}\right)}{\sigma},
$$

where $C_{7}=C_{7}\left(\sigma, \lambda+\frac{1}{2} C_{1}\right)$. We note that from (3.20) and (4.6)

$$
1-\left|u\left(z_{0}\right)\right|^{2} \leq 2 A_{2} e^{C_{1} / 4}\left(1-\left|z_{0}\right|^{2}\right),
$$

where $A_{2}=\sup _{\bar{B}} h(\geq 1)$. In consequence, it follows from (4.15) and (4.16) that

$$
\left|\sum_{j, \alpha=1}^{2}\left(\frac{\partial u^{j}}{\partial x^{\alpha}}\right)^{2}\left(z_{0}\right)\right|^{1 / 2} \leq \frac{2 A_{2} C_{7} e^{C_{1} / 4}\left(\lambda+\frac{1}{2} C_{1}\right)}{\sigma} .
$$

Since $z_{0}$ is an arbitrary point in $D, u$ is a Lipschitz map of $\bar{B}$ onto itself. This completes the proof.

Proposition 2. Let $u$ be a harmonic diffeomorphism, constructed in Theorem $2^{\prime}$, with $\operatorname{deg}(\varphi)=1$. Regard $u$ as a map $u \in C^{\infty}(B, B) \cap C^{0}(\bar{B}, \bar{B})$. Then there exists a positive constant $\delta_{1}=\delta_{1}(\varphi)$ such that

$$
J(u) \geq \delta_{1}^{-1}>0 \text { in } B .
$$

Remark 4. Combined with (3.28), (4.17) implies

$$
\left|\frac{\partial u}{\partial z}\right|^{2} \geq \delta_{1}^{-1}>0 \text { in } B .
$$

Lemma 10. Let $\overline{\widetilde{B}_{l_{j}}}\left(z_{j}\right)=\left\{z \in D ; \operatorname{dist}_{D}\left(z, z_{j}\right) \leq l_{j}\right\}$ for $j=1,2$. Suppose that a harmonic diffeomorphism $v: \overline{\widetilde{B}_{l_{1}}}\left(z_{1}\right) \rightarrow \overline{\widetilde{B}_{l_{2}}}\left(z_{2}\right)$ satisfies

$$
\operatorname{Vol}\left(v\left(\widetilde{B}_{s}\left(z_{1}\right)\right)\right) \geq \mu>0 \text { for some } s\left(0<s<l_{1}\right) \text {. }
$$

Then there exists a positive constant $\delta$ such that

$$
\|J(v)(z)\| \geq \delta^{-1} \quad \text { for } z \in \widetilde{B}_{l}\left(z_{1}\right),
$$

where $0<l<l_{1}, \delta=\delta\left(l_{1}, l_{2}, s, \mu, l\right)$, and $\|J(v)(z)\|$ stands for the normalized Jacobian of $v(z)$ by the metric $d s_{D}^{2}$.

Proof. Note that, since the isometry group of $D$ acts transitively on $D$, there exist a bounded positive $C^{0}$ function $f:[0,1] \rightarrow \mathbb{R}$ and a conformal diffeomorphism $w=w\left(z_{1}, l_{1}\right): \bar{B}(\subset \mathbb{C}) \rightarrow \widetilde{\widetilde{B}_{l_{1}}}\left(z_{1}\right)$ such that

$$
\|J(w)(z)\|=f(|z|) \text { for } z \in \bar{B},
$$


where $f$ is defined independently of $z_{1}$. Then, by applying Theorem 7.1 in [13] to the harmonic diffeomorphism $v \circ w: \bar{B} \rightarrow{\widetilde{\widetilde{B}_{l}}}_{l_{2}}\left(z_{2}\right)$, we obtain

$$
\|J(v \circ w)\| \geq \delta_{2}^{-1}>0 \text { on } B_{k},
$$

where $0<k<1$ and $\delta_{2}=\delta_{2}\left(l_{2}, s, \mu, k, f\right)$. Then, combining (4.21) with (4.20) yields (4.19).

Proof of Proposition 2. First, keeping the proof of Proposition 1 in mind, we shall show that there exists a positive constant $\sigma$ such that

$$
u\left(\widetilde{B}_{\sigma / 2}\left(z_{0}\right)\right) \supset \widetilde{B}_{1}\left(u\left(z_{0}\right)\right) \text { for all } z_{0} \in D \text {. }
$$

We note that, since $\varphi: S^{1} \rightarrow S^{1}$ is a $C^{4}$ diffeomorphism, there exist positive constants $r_{6}\left(0<r_{3}<r_{6}<1\right)$ and $C_{8}$

$$
0<C_{8}<\left|\frac{\partial(\arg (\Phi))}{\partial \theta}\right| \text { on } \bar{B} \backslash B_{r_{6}} .
$$

Choose a positive constant $\sigma$ large enough so that

$$
\frac{1}{4} \sigma+\min \left\{\log \frac{1}{4 A_{0}}, \log \frac{C_{9}}{2}\right\} \geq 1+\frac{1}{2} C_{1},
$$

where $C_{9}=\min \left\{1,\left(C_{8}\right)^{2} / 32\left(A_{2}\right)^{2}\right\}$. Let $z_{0}$ be a point in $D$ satisfying

$$
\log \left[\left(1+\left|z_{0}\right|\right) /\left(1-\left|z_{0}\right|\right)\right] \geq \sigma \text {. }
$$

Let $k_{1}=k_{1}\left(\left|z_{0}\right|\right), k_{2}=k_{2}\left(\left|z_{0}\right|\right)$, and $\eta=\eta\left(z_{0}\right) \quad(0<\eta<\pi / 2)$ be constants satisfying

$$
\begin{aligned}
0<k_{1}<\left|z_{0}\right| & <k_{2}<1 \\
\frac{1}{4} \sigma & =\log \left(\frac{1-k_{1}}{1-\left|z_{0}\right|} \cdot \frac{1+\left|z_{0}\right|}{1+k_{1}}\right)=\log \left(\frac{1-\left|z_{0}\right|}{1-k_{2}} \cdot \frac{1+k_{2}}{1+\left|z_{0}\right|}\right), \\
\cosh \left(\frac{1}{4} \sigma\right) & =\cosh ^{2}\left(\log \frac{1+\left|z_{0}\right|}{1-\left|z_{0}\right|}\right)-\cos \eta \sinh ^{2}\left(\log \frac{1+\left|z_{0}\right|}{1-\left|z_{0}\right|}\right) .
\end{aligned}
$$

Then, it follows from (4.25)-(4.27) that the truncated cone

$$
\mathscr{T}=\mathscr{T}_{0}\left(z_{0}, k_{1}, k_{2}, \eta\right)=\left\{z \in D ; k_{1}<|z|<k_{2}, \varangle_{0}\left(z, z_{0}\right)<\eta\right\}
$$

satisfies

$$
\mathscr{T} \subset \widetilde{B}_{\sigma / 2}\left(z_{0}\right)
$$

Let $W_{1}=\left\{z \in \overline{\mathscr{T}} ;|z|=k_{1}\right\}, W_{2}=\left\{z \in \overline{\mathscr{T}} ;|z|=k_{2}\right\}$, and $W_{3}=\{z \in$ $\left.\overline{\mathscr{T}} ; \varangle_{0}\left(z, z_{0}\right)=\eta\right\}$, respectively. Put $\partial \mathscr{T}=W_{1} \cup W_{2} \cup W_{3}$. It follows from (4.24) and (4.26) that for $z \in W_{1}$

$$
\begin{aligned}
\operatorname{dist}_{D} & \left(\Phi\left(z_{0}\right), 0\right)-\operatorname{dist}_{D}(\Phi(z), 0) \\
& =\log \left(\frac{1-|\Phi(z)|}{1-\left|\Phi\left(z_{0}\right)\right|} \cdot \frac{1+\left|\Phi\left(z_{0}\right)\right|}{1+|\Phi(z)|}\right) \geq \log \left(\frac{\left(1-k_{1}\right) h(z)}{\left(1-\left|z_{0}\right|\right) h\left(z_{0}\right)} \cdot \frac{1}{2}\right) \\
& \geq \log \left(\frac{1-k_{1}}{1-\left|z_{0}\right|} \cdot \frac{1+\left|z_{0}\right|}{1+k_{1}} \cdot \frac{1}{4 A_{0}}\right) \\
& =\frac{1}{4} \sigma+\log \left(\frac{1}{4 A_{0}}\right) \geq 1+\frac{1}{2} C_{1}>0 .
\end{aligned}
$$


Similarly, for $z \in W_{2}$

$$
\operatorname{dist}_{D}(\Phi(z), 0)-\operatorname{dist}_{D}\left(\Phi\left(z_{0}\right), 0\right) \geq 1+\frac{1}{2} C_{1}>0 .
$$

It is immediate from (4.23), (4.26), and (4.27) that there exists a constant $k_{3}$ $\left(0<r_{6}<k_{3}<1\right)$ such that for $k_{1}=k_{1}\left(\left|z_{0}\right|\right) \geq k_{3}$

$$
0<C_{8} \eta<\pi / 2, \quad 1-\cos \left(C_{8} \eta\right) \geq \frac{1}{2}\left(C_{8}\right)^{2}(1-\cos \eta) .
$$

Note that if $\left|z_{0}\right| \geq\left(\sqrt{2}-\left(2 A_{2}\right)^{-1}\right) /\left(\sqrt{2}+\left(2 A_{2}\right)^{-1}\right)$, then

$$
\frac{1+\left|z_{0}\right|}{1-\left|z_{0}\right|} \cdot \frac{1}{2 A_{2}}>1 \text {, }
$$

$$
\sinh \left(\log \left(\frac{1+\left|z_{0}\right|}{1-\left|z_{0}\right|} \cdot \frac{1}{2 A_{2}}\right)\right) \geq \frac{1}{4 A_{2}} \sinh \left(\log \frac{1+\left|z_{0}\right|}{1-\left|z_{0}\right|}\right)>0 .
$$

Put $k_{4}=\max \left\{k_{3},\left(\sqrt{2}-\left(2 A_{2}\right)^{-1}\right) /\left(\sqrt{2}+\left(2 A_{2}\right)^{-1}\right)\right\}$. Then, when $k_{1}=k_{1}\left(\left|z_{0}\right|\right) \geq$ $k_{4}$, it is verified from (4.6), (4.23), (4.27), and (4.31)-(4.33) that for $z \in W_{3}$

$$
\begin{aligned}
& \cosh \left(\operatorname{dist}_{D}\left(\Phi\left(z_{0}\right),\left|\Phi\left(z_{0}\right)\right| e^{i \cdot \arg (\Phi(z))}\right)\right) \\
&= \cosh ^{2}\left(\log \frac{1+\left|\Phi\left(z_{0}\right)\right|}{1-\left|\Phi\left(z_{0}\right)\right|}\right)-\cos \left[\arg (\Phi(z))-\arg \left(\Phi\left(z_{0}\right)\right)\right] \\
& \cdot \sinh ^{2}\left(\log \frac{1+\left|\Phi\left(z_{0}\right)\right|}{1-\left|\Phi\left(z_{0}\right)\right|}\right) \\
&= 1+\left[1-\cos \left\{\arg (\Phi(z))-\arg \left(\Phi\left(z_{0}\right)\right)\right\}\right] \\
& \cdot \sinh ^{2}\left(\log \frac{1+\left|\Phi\left(z_{0}\right)\right|}{1-\left|\Phi\left(z_{0}\right)\right|}\right) \\
& \geq 1+\left(1-\cos \left(C_{8} \eta\right)\right) \sinh ^{2}\left(\log \left(\frac{1+\left|z_{0}\right|}{1-\left|z_{0}\right|} \cdot \frac{1}{2 A_{2}}\right)\right) \\
& \geq 1+\frac{1}{2}\left(C_{8}\right)^{2}(1-\cos \eta)\left(\frac{1}{4 A_{2}}\right)^{2} \sinh ^{2}\left(\log \frac{1+\left|z_{0}\right|}{1-\left|z_{0}\right|}\right) \\
& \geq C_{9}\left[\cosh { }^{2}\left(\log \frac{1+\left|z_{0}\right|}{1-\left|z_{0}\right|}\right)-\cos \eta \sinh ^{2}\left(\log \frac{1+\left|z_{0}\right|}{1-\left|z_{0}\right|}\right)\right] \\
& \geq C_{9} \cosh \left(\frac{1}{4} \sigma\right) \geq \frac{1}{2} C_{9} e^{\sigma / 4}
\end{aligned}
$$

and then from (4.24)

$$
\operatorname{dist}_{D}\left(\Phi\left(z_{0}\right),\left|\Phi\left(z_{0}\right)\right| e^{i \cdot \arg (\Phi(z))}\right) \geq \frac{1}{4} \sigma+\log \frac{C_{9}}{2} \geq 1+\frac{1}{2} C_{1} .
$$

When $\log \left[\left(1+\left|z_{0}\right|\right) /\left(1-\left|z_{0}\right|\right)\right] \geq \sigma$ and $k_{1}=k_{1}\left(\left|z_{0}\right|\right) \geq k_{4}$, we get from (4.29), (4.30), and (4.34) that

$$
\operatorname{dist}_{D}\left(\Phi\left(z_{0}\right), \Phi(\partial \mathscr{T})\right) \geq 1+\frac{1}{2} C_{1} .
$$

Combining (4.35) with (3.19) then yields

$$
\operatorname{dist}_{D}\left(u\left(z_{0}\right), u(\partial \mathscr{T})\right) \geq 1 .
$$

It then follows from (4.28) and $u \in \mathscr{D}(D)$ that

$$
u\left(\widetilde{B}_{\sigma / 2}\left(z_{0}\right)\right) \supset u(\mathscr{T}) \supset \widetilde{B}_{1}\left(u\left(z_{0}\right)\right) \text {. }
$$


When $\log \left[\left(1+\left|z_{0}\right|\right) /\left(1-\left|z_{0}\right|\right)\right]<\sigma$ or $k_{1}=k_{1}\left(\left|z_{0}\right|, \sigma\right)<k_{4}$, we can choose $\sigma$ satisfying (4.22) by (4.6) and (4.26). Together with (4.36), this yields (4.22).

Now, owing to (4.14) and (4.22) we can apply Lemma 10 to $u \in \mathscr{D}(D)$. Then, there exists a positive constant $\delta_{3}=\delta_{3}\left(\sigma, \lambda+\frac{1}{2} C_{1}\right)$ such that

$$
\|J(u)\| \geq \delta_{3}^{-1}>0 \text { in } \widetilde{B}_{\sigma / 2}\left(z_{0}\right) .
$$

Since $\delta_{3}$ is independent of $z_{0}$, we obtain

$$
\left(\frac{1-|z|^{2}}{1-|u(z)|^{2}}\right)^{2}(J(u))(z) \geq \delta_{3}^{-1}>0 \quad \text { for } z \in D .
$$

Combining (4.37) with (3.20) and (4.6) then implies

$$
\begin{aligned}
(J(u))(z) & \geq\left(\frac{1-|u(z)|^{2}}{1-|z|^{2}}\right)^{2} \delta_{3}^{-1} \geq\left(\frac{e^{-C_{1} / 4}(1-|\Phi|)}{1-|z|^{2}}\right)^{2} \delta_{3}^{-1} \\
& \geq\left(\frac{e^{-C_{1} / 4} h(z)(1-|z|)}{1-|z|^{2}}\right)^{2} \delta_{3}^{-1} \\
& \geq\left(\frac{A_{1} e^{-C_{1} / 4}}{2}\right)^{2} \delta_{3}^{-1} \text { for } z \in D(\simeq B) .
\end{aligned}
$$

This completes the proof of Proposition 2.

\section{ENTIRE SPACELIKE CONSTANT MEAN CURVATURE SURFACES IN $\mathbb{L}^{3}$}

In this section, applying Theorem $2^{\prime}$ together with Proposition 1 and Remark 4 to the representation formula for spacelike surfaces in $\mathbb{L}^{3}$ [3], we shall construct entire spacelike constant mean curvature surfaces $M$ in $\mathbb{L}^{3}$, whose Gauss maps are harmonic diffeomorphisms of $M$ to $\mathbb{H}^{2}$ with $C^{4}$ diffeomorphisms $\varphi: S^{1}(\simeq M(\infty)) \rightarrow S^{1}\left(\simeq \mathbb{H}^{2}(\infty)\right)$ as prescribed boundary data.

We first review briefly relevant facts on Gauss maps of spacelike surfaces in $\mathbb{L}^{3}$. The Minkowski 3-space $\mathbb{L}^{3}$ is $\mathbb{R}^{3}$ equipped with Lorentzian metric $d s^{2}=d x^{2}+d y^{2}-d z^{2}$. The upper hyperboloid $\mathbb{H}^{2}=\left\{(x, y, z) \in \mathbb{L}^{3} ; x^{2}+\right.$ $\left.y^{2}-z^{2}=-1, z>0\right\}$ of future-directed unit timelike vectors in $\mathbb{L}^{3}$ is the hyperbolic plane with respect to the induced metric. The map $\mathscr{P}$ of $\mathbb{H}^{2}$ onto $D$ defined by

$$
\mathscr{P}:(x, y, z) \rightarrow\left(\frac{x}{1+z}, \frac{y}{1+z}\right)
$$

is an isometry. An immersed surface $M$ in $\mathbb{L}^{3}$ whose induced metric is Riemannian is called spacelike. The future-directed unit normal vectors of $M$ defines the Gauss map $G: M \rightarrow \mathbb{H}^{2}$. We shall also call the composition $\mathscr{P} \circ G: M \rightarrow D$ the Gauss map of $M$. The Gauss map of $M$ is harmonic if and only if the mean curvature of $M$ is constant [17].

With these understood, we prove

Theorem 3. Let $\varphi$ be a $C^{4}$ diffeomorphism of $S^{1}$ to $D(\infty)\left(\simeq S^{1}\right)$ with $\operatorname{deg}(\varphi)=1$. Then there exist a harmonic diffeomorphism $u \in \mathscr{D}(B, D) \cap$ $\mathscr{H}(\bar{B}, \bar{D})$ and an entire spacelike embedding $X: B \rightarrow \mathbb{L}^{3}$ with the following properties:

(1) $\left.u\right|_{S^{1}}=\varphi$. 
(2) $M:=X(B)$ has constant mean curvature 1, the Gauss map of $M$ is given by $u$, and the Gaussian curvature $K$ of $M$ satisfies $-1 \leq K \leq-a^{2}$, where $0<a=a(\varphi) \leq 1$.

(3) $X=\left(X^{1}, X^{2}, X^{3}\right)$ is given explicitly as

$$
\begin{aligned}
& X^{1}(z)=2 \operatorname{Re} \int^{z} \frac{1+\bar{u}^{2}}{\left(1-|u|^{2}\right)^{2}} \frac{\partial u}{\partial z} d z+c_{1}, \\
& X^{2}(z)=2 \operatorname{Re} \int^{z}(-i) \frac{1-\bar{u}^{2}}{\left(1-|u|^{2}\right)^{2}} \frac{\partial u}{\partial z} d z+c_{2}, \\
& X^{3}(z)=2 \operatorname{Re} \int^{z} \frac{2 \bar{u}}{\left(1-|u|^{2}\right)^{2}} \frac{\partial u}{\partial z} d z+c_{3}
\end{aligned}
$$

for $z \in B$, where $c=\left(c_{1}, c_{2}, c_{3}\right) \in \mathbb{R}^{3}$ and the integral being taken along an arbitrary path from a fixed point to the point $z \in B$.

(4) If $\varphi$ is not conformal, then the umbilical points of $M$ are isolated.

To prove Theorem 3, we prepare the following proposition.

Proposition 3 ([3]). Let $M$ be a simply connected Riemann surface, $H: M \rightarrow D$ be a nonvanishing real $C^{\infty}$ function on $M$, and $\Psi: M \rightarrow D$ be a nowhere antiholomorphic $C^{\infty}$ map of $M$ to $D$. Suppose $H$ and $\Psi$ satisfy the following differential equation:

$$
H\left(\frac{\partial^{2} \Psi}{\partial w \partial \bar{w}}+\frac{2 \bar{\Psi}}{1-|\Psi|^{2}} \frac{\partial \Psi}{\partial w} \frac{\partial \Psi}{\partial \bar{w}}\right)=\frac{\partial H}{\partial \bar{w}} \frac{\partial \Psi}{\partial w},
$$

where $w$ is a complex coordinate on $M$ compatible with its complex structure. Then there exists a spacelike immersion $X: M \rightarrow \mathbb{L}^{3}$ with the following properties: $\Psi$.

(1) The mean curvature of $M$ is $H$, and the Gauss map of $M$ is given by

(2) The induced metric $g$ on $M$ and the Gaussian curvature $K$ of $M$ are given by

$$
\begin{gathered}
g=\left(\frac{2}{H\left(1-|\Psi|^{2}\right)}\left|\frac{\partial \Psi}{\partial w}\right|\right)^{2}|d w|^{2} \\
K=H^{2}\left(\left|\frac{\partial \Psi}{\partial \bar{w}} / \frac{\partial \Psi}{\partial w}\right|^{2}-1\right)
\end{gathered}
$$

(3) $X=\left(X^{1}, X^{2}, X^{3}\right)$ is given explicitly as

$$
\begin{aligned}
& X^{1}(w)=2 \operatorname{Re} \int^{w} \frac{1}{H} \frac{1+\bar{\Psi}^{2}}{\left(1-|\Psi|^{2}\right)^{2}} \frac{\partial \Psi}{\partial w} d w+c_{1}, \\
& X^{2}(w)=2 \operatorname{Re} \int^{w} \frac{-i}{H} \frac{1-\bar{\Psi}^{2}}{\left(1-|\Psi|^{2}\right)^{2}} \frac{\partial \Psi}{\partial w} d w+c_{2}, \\
& X^{3}(w)=2 \operatorname{Re} \int^{w} \frac{2}{H} \frac{\bar{\Psi}}{\left(1-|\Psi|^{2}\right)^{2}} \frac{\partial \Psi}{\partial w} d w+c_{3},
\end{aligned}
$$

for $w \in M$, where $c=\left(c_{1}, c_{2}, c_{3}\right) \in \mathbb{R}^{3}$ and the integral being taken along an arbitrary path from a fixed point to the point $w$. 
(4) $(\partial \Psi / \partial \bar{w})\left(w_{0}\right)=0$ at a point $w_{0} \in M$ if and only if $w_{0}$ is an umbilical point of $M$.

Proof of Theorem 3. Let $u$ be a harmonic diffeomorphism with $\left.u\right|_{S^{1}}=\varphi$, which is constructed in Theorem $2^{\prime}$. In Proposition 3 , take $M=B, H=1$, and $\Psi=u$. It follows from (2.4), (3.29), and Remark 2 that $\Psi$ is nowhere antiholomorphic, $H$ and $\Psi$ satisfy (5.1), and that if $\varphi$ is not conformal, then the zeros of $\partial \Psi / \partial \bar{w}$ are isolated in $M$. Combining (3.28), (4.1), and (4.17) with (5.3) also yields $-1 \leq K \leq-a^{2}$. Hence assertions (1)-(4) hold.

It remains to prove that $M$ is entire in $\mathbb{L}^{3}$. Note that a complete spacelike surface in $\mathbb{L}^{3}$ is entire. Thus it suffices to show that $M$ is complete. But, substituting (3.20), (4.1), and (4.18) in (5.2), it is not hard to see that $M=$ $(B, g)$ is quasi-isometric to $D=\left(B, d s_{D}^{2}\right)$, and that $M$ is complete. This completes the proof.

\section{REFERENCES}

1. L.V. Ahlfors, Complex analysis, 3rd ed., McGraw-Hill, New York, 1979.

2. K. Akutagawa, The Dirichlet problem at infinity for harmonic mappings between Hadamard manifolds, Geometry of Manifolds, Academic Press, San Diego, 1989, pp. 59-70.

3. K. Akutagawa and S. Nishikawa, The Gauss map and spacelike surfaces with prescribed mean curvature in Minkowski 3-space, Tôhoku Math. J. 42 (1990), 67-82.

4. K. Akutagawa and A. Tachikawa, Nonexistence results for harmonic maps between noncompact complete Riemannian manifolds, Tokyo J. Math. 16 (1993), 131-145.

5. M. T. Anderson and R. Schoen, Positive harmonic functions on complete manifolds of negative curvature, Ann. of Math. (2) 121 (1985), 429-461.

6. P. Avilés, H. I. Choi, and M. J. Micallef, Boundary behavior of harmonic maps of non-smooth domains and complete negatively curved manifolds, Preprint.

7. H. I. Choi and A. Treibergs, New examples of harmonic diffeomorphisms of the hyperbolic plane onto itself, Manuscripta Math. 62 (1988), 249-256.

8. Gauss maps and spacelike constant mean curvature hypersurfaces of Minkowski space, J. Differential Geom. 32 (1990), 775-817.

9. P. Eberlein and B. O'Neill, Visibility manifolds, Pacific J. Math. 46 (1973), 45-109.

10. R. Hamilton, Harmonic maps of manifolds with boundary, Lecture Notes in Math., vol. 471, Springer-Verlag, Berlin and New York, 1975.

11. S. Hildebrandt, H. Kaul, and K. O. Widman, An existence theorem for harmonic mappings of Riemannian manifolds, Acta Math. 138 (1977), 1-16.

12. J. Jäger and $\mathbf{H}$. Kaul, Uniqueness and stability of harmonic maps and their Jacobi fields, Manuscripta Math. 28 (1979), 269-291.

13. J. Jost, Harmonic maps between surfaces, Lecture Notes in Math., vol. 1062, Springer-Verlag, Berlin and New York, 1984.

14. J. Jost and H. Karcher, Geometrische Methoden zur Gewinnung von a-priori-Schranken für harmonische Abbildungen, Manuscripta Math. 40 (1982), 27-77.

15. P. Li and L.-F. Tam, The heat equation and harmonic maps of complete manifolds, Invent. Math. 105 (1991), 1-46.

16. $\_$, Uniqueness and regularity of proper harmonic maps, Ann. of Math. 137 (1991), 167-201.

17. T. K. Milnor, Harmonic maps and classical surface theory in Minkowski 3-space, Trans. Amer. Math. Soc. 280 (1983), 161-185.

18. R. Schoen and S.-T. Yau, On univalent harmonic maps between surfaces, Invent. Math. 44 (1978), 265-278. 
19. A. Tachikawa, Harmonic mappings from $\mathbb{R}^{n}$ into a Hadamard manifold, J. Math. Soc. Japan 42 (1990), 147-153.

20. A. Treibergs, Entire spacelike hypersurfaces of constant mean curvature in Minkowski space, Invent. Math. 66 (1982), 39-56.

21. P. Li and L.-F. Tam, Uniqueness and regularity of proper harmonic maps, II, Indiana Math. J. 42 (1993), 591-635.

Department of Mathematics, Shizuoka University, Shizuoka 422, Japan

E-mail address: k-actaela. shizuoka.ac.jp 